\title{
MIDAS: A Software Framework for Accommodating Heterogeneous Interaction Devices for Cloud Applications
}

\author{
Euijai Ahn, Kangyoon Lim, and Gerard Jounghyun Kim \\ Digital Experience Laboratory \\ Korea University, Seoul, Korea \\ \{saintpio, nandalky, gjkim\}@korea.ac.kr
}

\begin{abstract}
Even though the computational power and storing capacities of mobile computing platforms have recently increased dramatically, so have the needs of mobile applications. With the advent of the cloud computing and wireless network technology, the mobile device finds itself as an ideal candidate as a multi-purpose interaction client device instead of as a standalone computing station. In the line of such a trend, we present a software framework (called MIDAS) that enables an application to lend itself to many different types of interaction methods (namely, sensing and display) and accommodate users with variant client devices without platform specific coding. The paper discusses the requirements and the design of the software architecture, and in addition, demonstrates its effectiveness with several case studies.
\end{abstract}

Keywords: Cloud based Interaction, Ubiquitous Interaction, Multimodal Interaction, Software Framework.

\section{Introduction}

Cloud computing, high speed wireless communication and smart phones have the potential to realize the true form of ubiquitous computing as conceptualized by Weiser nearly two decades ago [1]. The smart phone (as a broadband wireless communication device) based cloud computing allows the instant access of rich information and high quality service anywhere, and in addition, the smart phone (as a rich media device) can also serve as the medium through which the user interact efficiently and naturally. Thus we can easily imagine a future situation where users with variant smart phones (or hand-held devices with similar capabilities) accomplishing a given application task (e.g. shooting an opponent in a first person shooting game), but in different interactional forms (e.g. using the button, touchscreen, or voice). This may stem simply from the differences in the device processing, sensing and display capabilities (e.g. button-less device forcing the user to use the touchscreen) or simply from personal choice (e.g. preferring the use of button over touchscreen due to finger occlusion of the screen). 
Traditionally, to accommodate a situation as described above, either separate client programs are developed for (or ported to) different devices (also because different devices often run on different system softwares and operating systems) enforcing a particular interface most suited for the given device, or a "large" client program is developed to cover all possible interaction possibilities. Practically, this has caused application services (cloud or local) to be compatible to only a small family or brand of devices and leaving no choices for the users in terms of interaction possibilities. Note that today's smart phones are not only increasingly equipped with various types of sensors, but can process computationally intensive recognition algorithms in real time (but only to a degree), not to mention meditate cloud based solutions. As such, user demands and expectations are higher with regards to the degree of interaction possibility.

In the line of such a need, we present a software framework, called MIDAS (Mixing and matching heterogeneous Interaction Devices to Applications and Services), that enables an application to lend itself to many different types of interaction methods (namely, sensing and display) and accommodate users with different client devices in a flexible manner (e.g. dispensing with the separate device dependent implementations). Through the proposed framework, the application server and interaction client device, upon connection, exchanges information regarding the generic interaction events (in the application) and the interaction methods/capabilities available in the client. Then the interaction events and the client interaction methods are mapped dynamically to allow the client to enact the application and receive output.

This paper is organized as follows. After comparing our work with other related research, the paper will first discuss the requirements and the design of the overall software architecture of MIDAS. Then in later sections, we demonstrate and present effectiveness of MIDAS with several use cases. Then we conclude the paper with a summary and directions for future work.

\section{Related Work}

The objective our work bears much similarity to concepts like migratory and plastic interfaces in the context of ubiquitous computing. Migratory interfaces are those that operate on changing operating platforms or interaction resources (e.g. a control panel interface for a whiteboard "migrating" and rendered on a connecting PDA). Plasticity further requires the migrating interface to preserve the usability as much as possible by adaptation to the specific operating platform (e.g. the control panel lay out changed according to the size of the PDA) [2]. Another early approach to realize multi-device interaction is the redirection technique [3][4] in which input events or content output may be sent to another collaborating/connected device. In all cases, separate platform specific implementations or compilation processes are needed.

For a more general and flexible migration and plasticity, several researchers have been working on a larger software framework or infrastructures. For example, Cameleon-RT and BEACH (as realized in the I-AM [5] and i-LAND [6]) are examples of such software architecture/infrastructures in which multiple interaction devices 
could be managed and interact with the given application in the most suited way. The middleware enables a "generically" described interactive application to build their own "view" for a given physical interaction platform. TERESA [7] is an authoring tool for "One Model, Many Interfaces" [8] type of applications. With the help of TERESA, different user interface codes can be conveniently generated from abstract user interface descriptions. However, these architectures serve to avoid separate implementations for different interaction devices at compile time. That is, only devices whose capabilities are known ahead of time can be accommodated.

Migratory or plastic architectures inevitably are distributed since the middleware components may reside among different "client" physical platforms [5]. In particular, the main application (possibly on the server or in the cloud) may be purely functional with its interface described generically or in abstract form to be interpreted and customized on the client side. One such approach is the SEESCOA component system [9] where abstract UI described in XML is interpreted to compose user interfaces at run time (by the adaptation/rendering components). Mark up languages for interactive web and cloud applications that describe UI objects, and interpreted and realized by different platform browsers, operate on similar principles [10][11]. Gilroy et al. has developed a client middleware that adapts application's "presentation styles" (expressed in an XML format specification document) most suited for a given device at run time. This way, the menus, form-fills, dialogue GUIs are adjusted and presented according to the device capabilities and thus high usability can be expected [12]. The $\mathrm{X}$-Window system allows an application, through the $\mathrm{X}$ protocol, to interact with any device that runs the $X$ server which handles the user interaction [13]. X server only supports the standard keyboard and mouse interface.

\section{MIDAS Framework}

\subsection{Main Objectives}

In this section, we describe the details of the software framework, called MIDAS (Mixing and matching heterogeneous Interaction Devices to Applications and Services), especially its main objectives and operating mechanism. First, we illustrate what we hope to achieve through MIDAS through a simple scenario.

Michael has a feature phone (on which the MIDAS interaction client software is installed). He goes to a movie theater. At the theater, there is a public display kiosk displaying previews of the feature movies. The kiosk has no external input devices, because it is designed to serve multiple people at once and the display needs to be protected from the public from possible abuse. So Michael uses his phone to connect to the application and interacts with the buttons to view specific movie information and selections for the movie and the seat. At the same time, Michelle comes along with her latest smart phone equipped with voice recognition software. She, too, connects to the same application and uses the touch screen and voice recognition to select the movie and seats. Michael goes to the nearby snack shop and notices a long waiting line. This time, he connects to the snack menu application using MIDAS, 
browses the choices, and makes an order, again using the buttons on his feature phone.

In this scenario, some of the important objectives of MIDAS are highlighted. One is the accommodation of different models of interaction devices dynamically (e.g. feature phone, smart phone, hand-held media devices, etc.). Different models of smart phones differ in the display sizes, types of sensors, and processing power and can affect how the user wants to interact with the application. The accommodation must be "dynamic" in the sense that the middleware should adapt to the different device capabilities at run time (at least to some degree). Another related but distinct objective is to allow different interaction methods. For example, in the above scenario, Michelle can choose to use the voice recognition or touch screen (or both) to interact with her latest smart phone. Finally, an important but less emphasized objective of MIDAS is the ability to coordinate interaction and share single application space among multiple users. MIDAS allows multiple users but the support for resource sharing and collaboration is only rudimentary.

The scenario also illustrates the larger context for which MIDAS is to be used for, that is, a ubiquitous computing environment in which there are dichotomous re-sources combined dynamically to enable a wholistic computational service and inter-action experience. The environment will contain high powered and fidelity computing, sensing and display resources (e.g. cloud, large location specific servers, high resolution display, sensor network) for things like processing large volume data and computationally intensive services for many users, while the individuals are expected to the lesser (e.g. mobile devices, wearable devices, hand-held media devices) mainly for interactional and personalization purpose.

\subsection{Operating Mechanism and Architecture of MIDAS}

Before presenting the overall architecture of MIDAS, we first go over the operating mechanism to help the readers understand the composition of MIDAS.

Server and Client Connection and Loading of MIDAS Modules. MIDAS, as a middleware to achieve the aforementioned objectives, is distributed among the servers and interaction clients. Aside from the MIDAS itself (running on the application server or client), there is a separate "Connection and Arbitration" server (dubbed "CAS" hereafter) that manages the connection between a number of available MIDAS-based applications (servers) and interaction devices. The application servers register themselves with the CAS to announce its existence and availability to the potential client devices. On the other hand, through the CAS, the MIDAS client devices can search for available MIDAS application servers that they can connect and interact to use. To reiterate, we are envisioning that there can be number of different cloud/ubiquitous applications available accessible with one's client interaction device. Once the client interaction device (i.e. user) chooses one of the registered available application service, it requests a connection to it through the TCP/IP. 
In addition to this aforementioned role, the CAS also takes the role to relay the interaction capabilities of the connected server and client device. Both the server and client maintain a file describing its respective interaction capabilities. For instance, a particular version of a MIDAS server might be equipped to handle 10 different interaction methods, while the client device is only capable of two of them. The interaction methods acceptable to the application server and those that are available on the client are all described using an XML style "Interaction Resource" file, prior to the execution.

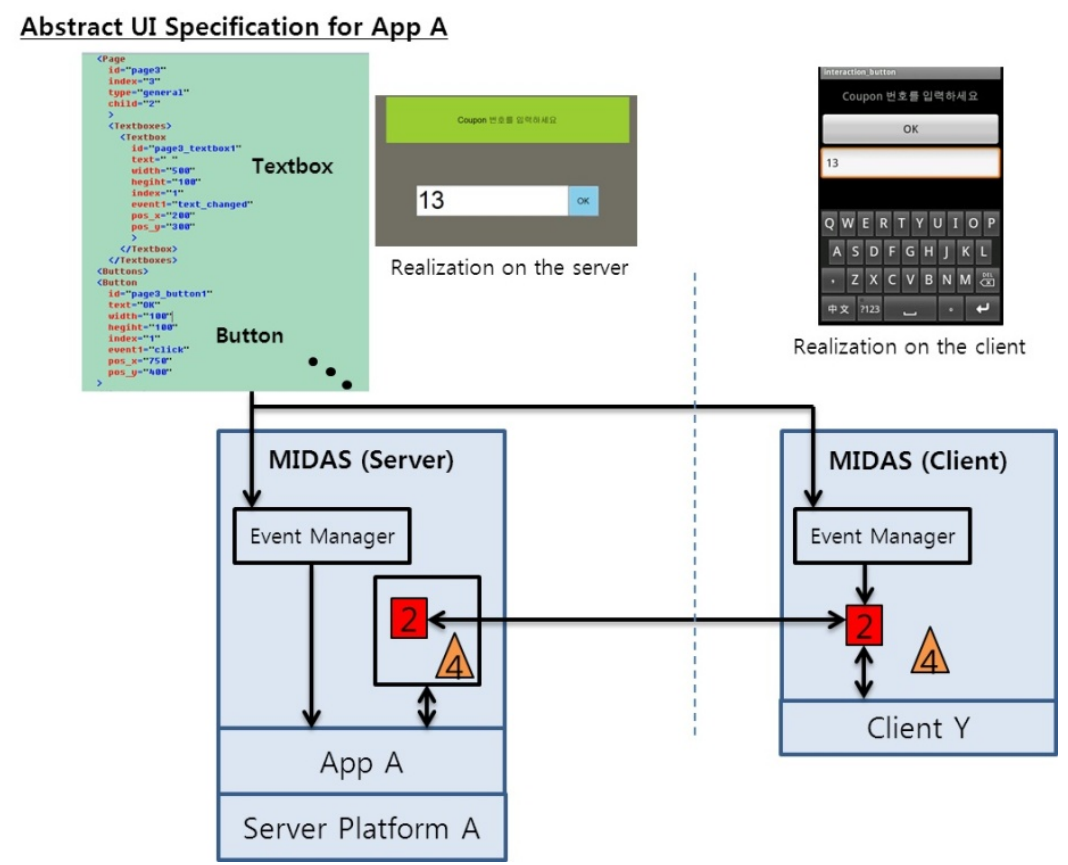

Fig. 1. Interpreting the application's user interface specification and sharing the event identification codes for mutual understanding between the server and client

User Interface Description and Management. Once the connection is established and proper modules all loaded, all possible "raw" client interaction events (e.g. touch screen click on a smart phone) must be mapped to the appropriate events in the application. In order to establish this mapping, an explicit application user interface description file is used. Figure 1 illustrates this process. An XML based user interface specification for the application is shared and interpreted by the server and client. UI objects are instantiated on the server and client (if necessary) by the "Event Manager" module on the respective side. These "Event Managers" (which are part of MIDAS server or client) are tailored for a given platform, and thus, the UI objects will have different look and feel accordingly (see Figure 1). During this step, the UI objects and 
the raw interaction events are assigned consistent indexed codes for identification on the either side. This way, any MIDAS client can be used as interaction device and no application specific modules are needed on the client side.

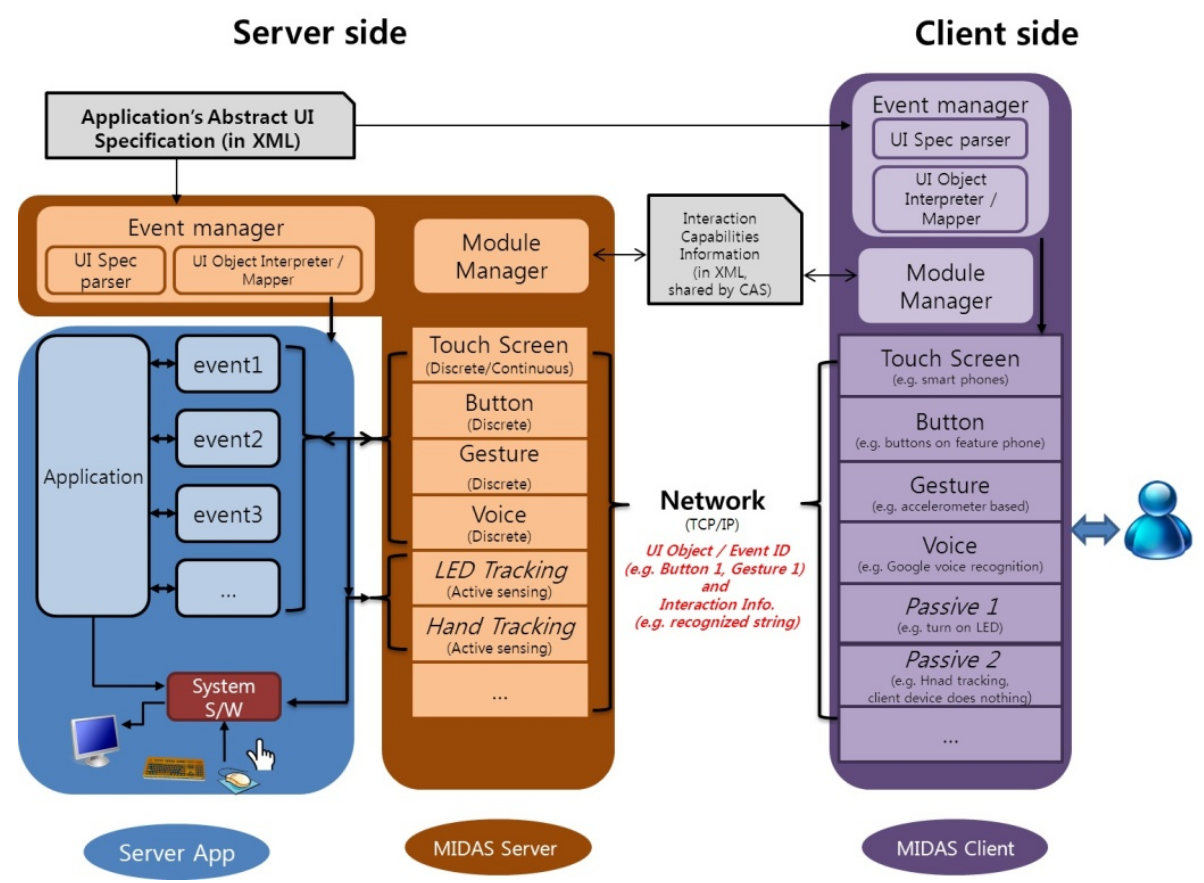

Fig. 2. The overall architecture of MIDAS

Overall Architecture. Consolidating all the mechanisms as explained in the previous sections, Figure 2 shows the overall architecture of MIDAS. The module manager on each side, load the proper MIDAS interaction information communication modules upon connection via CAS and by exchanging the mutual interaction capabilities (see previous subsection). The Event Managers on the respective sides also generate a consistent event mapping structure exchanged over the network so as for the application to invoke the right handler.

There are various MIDAS "modules" for different predefined types of interaction methods. They can be roughly classified into three groups: (1) discrete event type (e.g. button press, gesture recognition), (2) continuous input (e.g. cursor movement), (3) mixed type (e.g. text box with string input), (4) no-op (e.g. when the client device is used only as a physical target. For example, the touch screen on the client side would generate both "discrete" (e.g. button press) and "continuous" (e.g. finger location) events and data. The discrete events would be directly relayed to the application, while the continuous data can be "adjusted" or "filtered" and rather relayed to the server's system software to emulate mouse behavior. 


\section{$4 \quad$ Typical Use Cases}

\subsection{Replicating Server's User Interface on the (Multiple) Clients}

One of the most typical uses of MIDAS would be to replicate the server application user interface (or interaction objects) on to the client device for private interaction. In order for this to happen, the user interface of the server application is first described declaratively (using the XML based UI configuration specification format) as already explained. In run time, the UI objects described in the UI configuration file, would be created (and displayed if e.g. a public display was available). The client, upon connecting to the server, will first load proper middleware modules (for mapping the client's various interaction events to those on the server side). Then the same UI configuration file received from the server is parsed and interpreted to be replicated on the client side. Replication of the server's UI components on the client side would ideally include an "adaptation" process for UI plasticity. Currently, MIDAS has only very limited such capability. Figure 1 shows an example with a simple application interface consisted of a text box, label and button replicated on the client device.

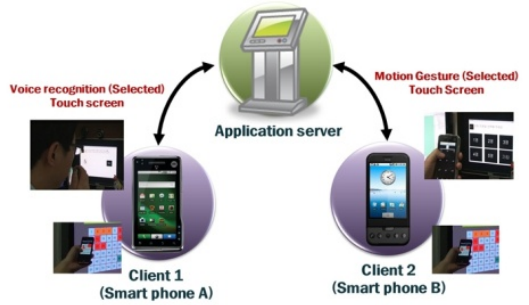

(a)

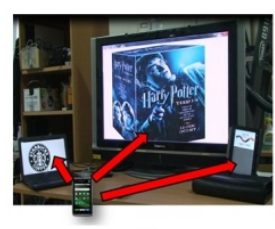

(b)

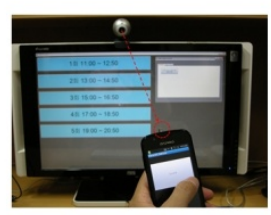

(c)

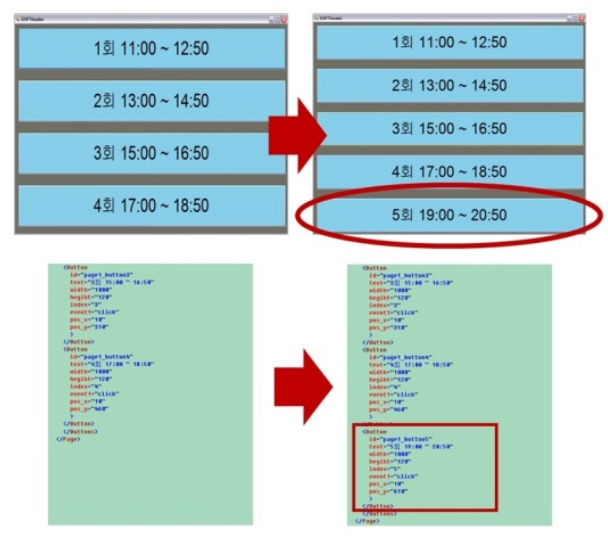

(d)

Fig. 3. Four representative use cases of MIDAS: (a) one application, many interfaces, (b) one client device connecting to many servers (e.g. coffee shop application (left), movie theater application (middle), subway application (right)), (c) user interaction processing distributed among the server and client (e.g. tracking by server, and touch processing by phone), (d) simple change of the user interface through modification of the UI specification file.

\subsection{One Application, Many Interfaces}

Extending to the first style of interaction, MIDAS naturally allows many modes of interaction according to the client's interaction capability. In the first example, the client device could have had both a touch screen or voice recognition capability to generate an event to select a menu item, and similarly so, for different clients who 
may possess different capabilities. When the client has more than one method available for generating a particular event, MIDAS first asks explicitly the user to select the method (e.g. touch screen or voice recognition). For instance, a client that can recognize 12 gestures might generate 12 events to the application events (e.g. Gesture 1 to App. Event 1, Gesture 2 to App. Event2 and so on). By default, the mapping is mechanical and arbitrary (e.g. in the implicit order of appearance in the UI specification file), but also user configurable through explicit specification in the XML UI specification file (See Figure 3(a)).

\subsection{One Client Middleware, Many Applications}

With MIDAS framework, the interaction client needs only the middleware (i.e. no application specific program) in order to connect and use any applications developed and running under the MIDAS server middleware. The same mapping mechanism and protocols are used for a client device to make input and receive output from a number of application servers as envisioned in the scenario described in Section 3.1 (see Figure 3(b)).

\subsection{Distributed UI Infrastructure}

MIDAS allows different distributions of user interaction processing, at one extreme, where the server is responsible for everything and at another, the client. For instance, one can imagine a public server with the device tracking and gesture recognizer that tracks and recognizes the movement of the LED light installed on the client device (See Figure 3(c)). In this case, client device does nothing computationally, except for registering itself with the server and letting it know that it has the compatible LED (used as the fiducial in tracking). We can suppose another case where the client device is equipped with an acceleration sensor and producing a sequence of acceleration value profiles, filtered conveyed to the application for recognition only. Finally, the tracking and recognition may be done entirely on the client device and only mapping the gesture event to the server. MIDAS's distributed and flexible protocol allows different load sharing of the UI processing (both computation and sensing wise) and thus makes it possible to accommodate devices with a variety of capabilities

\subsection{Ease in the Application Development and Maintenance}

Since MIDAS middleware separates the interaction "methods" (e.g. gesture recognition) and "events" (e.g. "Button 1" pressed) that are realized by the methods, the application development is much easier in that the developer needs not to worry about the various interaction methods available in different client device platforms (see Figure 3(d)). Since abstract user interface specification is used, in which UI object attribute information is included (to some extent), changing UI look and feel (e.g. GUI layout, fonts, sizes) is also possible without explicit recompilation. 


\section{$5 \quad$ Discussion and Conclusion}

In this paper, we presented MIDAS, a user interface middleware (or server), that is designed to accommodate different client interaction devices for a given application. Migration and plasticity for multi-device interaction is needed because the computing service is again truly becoming centralized and ubiquitous (but transparently so, e.g. through the cloud), and on the other hand, the client devices are ever so diversifying in terms of their processing, sensing and display capabilities. In particular, the mobile clients will employ different interaction styles and input methods depending on the operating environment and conditions and application contexts [14]. MIDAS borrows several concepts such as abstract user interface interpretation, distributed event processing, and decoupling of interface methods and abstract application events.

MIDAS is an improvement to the latest approaches in migratory or plastic interface in mainly two ways. First, it focuses on supporting a variety of input methods. Prior approaches mostly have dealt with customizing application's output on different platforms. However, MIDAS can also be extended further to support more plasticity for platform output presentation as well. While output format customization would rely on conforming to certain adaptation rules (e.g. if screen size is reduced, reduce the button size in the same proportion), supporting a wide variety of and comprehensive input methods hinges on categorizing the input type, e.g. discrete event and data, continuous data, etc. and engineering a support structure that is as light and compact as possible.

Secondly, MIDAS is flexible in the distributed composition of UI processing. The paper has presented two typical examples, one in which the server senses and recognizes the user with the client essentially doing nothing computationally and another where, e.g. the voice is recognized on the client device (actually by connecting to yet another cloud server).

While MIDAS, as currently implemented, offers basic components for dynamically leveraging the environment and individual resources (i.e. computers, sensors, displays), it still falls short to be of complete. As already indicated, the output plasticity is either only ad-hoc or rudimentary at best. While multiple users can be supported, concurrency control is not possible yet. The scalability is in question; that is, architecturally, we would like to design a general purpose middleware to accommodate a comprehensive input methods rather than adding new MIDAS modules to each new interaction methods. As alluded above, the crux will be in generalizing different input methods according to their event and data types, and designing a general yet flexible software architecture. Despite its current shortcomings, we believe that the proposed framework is one way to realize the seamless computing environment of the future where processors, data, sensors, and displays are distributed ubiquitously in our everyday living environment.

Acknowledgement. The research described in this work was supported by the Korean Ministry of Knowledge Economy`s Strategic Technology Laboratory Program. 


\section{References}

1. Weiser, M.: Hot topics-ubiquitous computing. Computer 26(10), 71-72 (1993)

2. Calvary, G., Thevenin, D.: A unifying reference framework for the development of plastic user interfaces. In: Nigay, L., Little, M.R. (eds.) EHCI 2001. LNCS, vol. 2254, pp. 173-192. Springer, Heidelberg (2001)

3. Elmqvist, N.: Distributed User Interfaces: State of the Art. In: Gallud, J.A., et al. (eds.) Distributed User Interfaces: Designing Interfaces for the Distributed Ecosystem. HumanComputer Interaction Series, pp. 1-12. Springer (2011)

4. Biehl, J.T., Bailey, B.P.: ARIS: an interface for application relocation in an interactive space. In: Graphics Interface 2004. Canadian Human-Computer Communications Society, pp. 107-116 (2004)

5. Balme, L., Demeure, A., Barralon, N., Calvary, G.: Cameleon-rt: A software architecture reference model for distributed, migratable, and plastic user interfaces. In: Markopoulos, P., Eggen, B., Aarts, E., Crowley, J.L. (eds.) EUSAI 2004. LNCS, vol. 3295, pp. 291-302. Springer, Heidelberg (2004)

6. Tandler, P.: Software infrastructure for ubiquitous computing environments: Supporting synchronous collaboration with heterogeneous devices. In: Abowd, G.D., Brumitt, B., Shafer, S. (eds.) UbiComp 2001. LNCS, vol. 2201, pp. 96-115. Springer, Heidelberg (2001)

7. Mori, G., Paterno, F., Santoro, C.: Design and development of multidevice user interfaces through multiple logical descriptions. IEEE Transactions on Software Engineering 30(8), 507-520 (2004)

8. Paterno, F., Santoro, C.: One model, many interfaces. In: Kolski, C., Vanderdonckt, J. (eds.) Computer-Aided Design of User Interfaces, vol. 3, pp. 143-154. Kluwer Academic Publishers (2002)

9. Luyten, K., Vandervelpen, C., Coninx, K.: Migratable user interface descriptions in component-based development. In: Forbrig, P., Limbourg, Q., Urban, B., Vanderdonckt, J. (eds.) DSV-IS 2002. LNCS, vol. 2545, pp. 44-58. Springer, Heidelberg (2002)

10. XHTML2 Working Group Home Page, http: / /www.w3 . org/MarkUp

11. XAML Overview (WPF), http://msdn.microsoft.com/en-us/library/ ms 752059 .aspx

12. Gilroy, S.W., Harrison, M.D.: Using interaction style to match the ubiquitous user interface to the device-to-hand. In: Feige, U., Roth, J. (eds.) DSV-IS 2004 and EHCI 2004. LNCS, vol. 3425, pp. 325-345. Springer, Heidelberg (2005)

13. X Window System, http://en.wikipedia.org/wiki/X_Window_System

14. Terrenghi, L., Lang, T., Lehner, B.: Elastic mobility: stretching interaction. In: 11th International Conference on Human-Computer Interaction with Mobile Devices and Services. ACM (2009) 